\title{
Fusion algorithms and high-performance applications for vehicular cloud computing
}

\author{
James J. Park ${ }^{1}$
}

CC Springer Science+Business Media, LLC, part of Springer Nature 2018

\section{Introduction}

Connected car services have become more popular due to the smartphone car service including Android Auto, Apple CarPlay and so on. These services provide Internet services, vehicle control \& monitoring applications, navigation and multimedia service for comfortable, convenient driving. On the other hand, mobile cloud computing is a combination of technology including cloud computing, mobile computing, and wireless network to provide various IT services and rich application. Mobile cloud computing can be applied to various domains including mobile learning, smart vehicular, healthcare and wellness, and others.

Vehicular Cloud Computing (VCC) has emerged recently, receiving great attention in ICT environments. The aforementioned services and technologies are combined into VCC for smart, intelligent driving experience. Given the advancements in wireless communication technology, it can extend the advanced automobile technologies using cloud computing services for better road safety and secure intelligent urban traffic systems. VCC is a new hybrid technology that consists of various network combinations such as mobile ad hoc networks, wireless sensor networks, vehicular ad hoc networks, and cloud computing. There may be many issues in realizing and providing smart services, and it has been paid enormous attention.

Meanwhile, fusion algorithms and high-performance applications including new technologies, concepts, or analyses, reports on experiences and experiments on implementation and application of theories, and tutorials on new trends are needed in VCC research fields. For such reasons, this special section seeks to give an overview of

$凶 \quad$ James J. Park

jhpark1@seoultech.ac.kr

1 Seoul National University of Science and Technology, Seoul, South Korea 
state of the art as well as issues and solution guidelines for VCC. It will also provide a compelling panorama of current research efforts, which is widely inherent in topics of high interest for the fusion algorithms and high-performance applications for VCC.

This special section solicited innovative ideas and solutions in all aspects revolving around fusion algorithms and high-performance applications for VCC. The general scope of this issue covers the fusion algorithms, technologies and scenarios, highperformance applications, and technologies for VCC. It also includes new approaches for VANET, CPS and IoT for VCC, Inter- \& Intra-Vehicle communication technologies, high-accuracy positioning technology, remote vehicle control, intelligent services for connected car, security and safety issue for VCC, and others. As a result, with careful consideration, this special section selects and publishes excellent research articles from a large number of contributed papers.

\section{Related works}

Xu et al. [1] introduced a shareable keyword search scheme based on ID-based encryption. The new scheme enables users to search in data owners' shared storage while preserving the privacy of data, which is suitable for the cloud computing environment. In this paper, data owners can share their document with their friends, who in turn can search in the data owner's storage while preserving the owner's privacy. The experiment result provides better efficiency compared with other related works, and security proofs show the correctness and soundness of the scheme.

Nkenyereye et al. [2] proposed a novel protocol for secure vehicle traffic data dissemination and analysis in vehicular cloud computing wherein the privacy of the vehicles and their generated message is ensured through pseudonym techniques. They use anonymous credentials to ensure the authorization of demanding vehicles. An ID-based signature is applied to ensure the authenticity of the vehicle for a given pseudonym. The efficient verification of the signature of the message and the discarded vehicle are made through the batch cation technique and pseudonymous revocation list, respectively.

As the massive data market grows explosively, cloud storage systems face significant challenges in maintaining large data volumes [3]. Due to the diversity and volume of data sets, the demand for new, resilient storage systems is increasing. While replication is widely used as a means of improving the availability and throughput of storage systems, some important features of replication have yet to be discovered. Data classification and block-based replication are promising in terms of improving the efficiency of cloud storage. Chen et al. [4] proposed an elastic, efficient file storage called E2FS, which can dynamically expand and shrink storage systems based on the real-time demands of large data applications. According to their experiments, E2FS can outperform HDFS while ensuring the performance of large data applications.

Munoz and Villalba [5] presented a new method of mapping web vulnerability classification based on diverse automatic scanners. The most common tool for analyzing vulnerabilities in Web applications is the automatic scanner. Note, however, that it is difficult to compare a particular scanner with the best - or at least the most appropriate-scanner to detect a particular vulnerability. It is important to define some 
evaluation criteria to evaluate scanner capabilities. This paper defines several evaluation criteria to estimate scanner functionality with flexibility, from the past to the latest classification techniques. The key of the proposed method is to reduce each vulnerability to a small, unique group of keywords representing the vulnerability and search for relations on the Internet.

Lim et al. [6] proposed an efficient solution to traffic control management for the intersection, which is one of the key issues in the research and development of ITS and its applications, based on VANET [7] and VCC [8]. It proposes an algorithm for the traffic control management for intersection in the context of VANET. The solution does not utilize a broadcast mechanism but works with point-to-point communication. The proposed algorithm solves the mutual exclusion problem for intersection traffic control using point-to-point communication, which is designed as an efficient distributed mutual exclusion algorithm based on the lead vehicle on each lane at the intersection; a local vehicle information exchange mechanism that supplements the mutual exclusion algorithm was also developed for the intersection traffic control problem.

Ji et al. [9] proposed a phased array image method based on compression sampling and data fusion for wireless structure damage monitoring. Structural health monitoring (SHM) is an important area of research in wireless sensor networks. Note, however, that the damage identification of SHM has problems with detection accuracy and network traffic. To solve this problem, the proposed method first obtains the compressed measurements in the sparse region of the damaged signal, performs data fusion with the Bayesian, and finally reconstructs the structural damage signal. Experimental results show that the proposed method can reduce the data transmission cost and balance network energy in SHM.

Software as a service (SaaS) technology in ubiquitous cloud computing uses customer relationship management (CRM) to enhance the quality of service for customers. Note, however, that traditional CRM is different from SaaS CRM since companies do not need to invest in machinery, equipment, and manpower to maintain the operation of CRM systems, which is suitable for various enterprises. Based on the research framework of the Stimulus-Organism-Response model [10], the cloud CRM projects based on multi-criteria decision-making (MCDM) analysis tool do not require prior assumptions to explore the weights, performances among project risks, project management, and organizational performance. The most representative MCDMs are the DEMATEL-based analytical network process (DANP) [11] and the VIKOR (VlseKriterijumska Optimizacija I Kompromisno Resenje) technique [12].

Chen et al. [13] studied a discussion on the relevance of cloud CRM project risk management and performance. The empirical results of this study show that the largest criterion for relative weight is mainly connected to the risk dimension, which represents the project risk assessment of the experts. In addition, cloud CRM experts and companies announced that the financial performance should improve during the process of a CRM project. This result means that companies implementing cloud CRM projects must pay attention to project risk and financial performance management.

Ramadoss et al. [14] proposed a novel approach to non-intrusive, transaction-aware filtering during enterprise modernization. The enterprise based on this approach can utilize the existing business knowledge and filter out the transaction characteristics, which will be useful for further business analysis and decision making by generating 
and deducing the documentation on legacy systems. They use transaction logs as input and focus on maximizing the information value and minimizing the size of the data collected. It has the advantage of being non-intrusive and applicable to a wide range of systems on cloud computing.

Lee et al. [15] introduces a new type of problem called remote data authenticity problem for stream data that are acquired and stored remotely. They propose a method for generating metadata for authenticating stream data using secure data collection and signature extraction modules. To share the encryption key, it generates the signature and exchange messages with signatures. The module of the proposed method establishes a secure communication channel with the verifier that attests to the authenticity of the remote data. The proposed method can remotely acquire and successfully detect loss and modification of the stored stream data.

The one critical issue analyzing ubiquitous networks is to understand the internal structure of the networks. In spite of various approaches that address structure mining in the graph, these approaches unfortunately cannot cope with the big graph generated by large-scale streaming data [16]. Hao and Park [17] presented cSketch, an efficient mining framework that enables capturing the cliques quickly and aims to mine the structure from the big graph. First, the input graph stream is summarized into another simplified graph stream via a hash function. Then, the FCA (formal concept analysis) theory and the previously defined k-equiconcept are adopted to discover the k-cliques from it. According to proof, the proposed framework demonstrates feasibility and effectiveness.

Kim [18] proposed a load balance scheme with Loadbot agent in IoT. This paper proposes a load balance scheme through Loadbot, which measures network load and processes the structural configuration by analyzing a large amount of user data and the network load by applying the deep belief network method. The proposed scheme uses the neural load prediction algorithm based on deep learning's Q-learning method to achieve efficient load balancing in IoT. The experiment results show the effectiveness of the proposal by comparing with previous researches.

Min et al. [19] proposed an optimization method that integrates resource allocation scheduling and traffic routing to improve data transmission speed by increasing network utilization in STDMA-based multi-hop wireless mesh networks. They also proposed JRS-S and JRS-M algorithms that simultaneously use path discovery and resource allocation to maximize the capacity of wireless mesh networks in cloud computing. The proposed algorithm for each flow uses a cross-layer design method based on numerical modeling to control data scheduling adaptively at the link layer and find a high data rate path with minimum interference at the network layer.

The efficiency of large data transfers from remote cloud platforms is one of the critical issues. Recently, there has been massive research interest regarding the utilization of big data via remote cloud platforms [20,21]. Lee et al. [22] measured throughput and analyzed the measurement according to L2 tuning factors and IP levels, including kernel parameter turning. They discussed improving throughput performance by setting an appropriate window size that takes into account the buffer size used in the experiment. L2 dedicated equipment has recently been installed in dedicated network equipment. It is important to optimize tuning factors to improve performance, because the performance of the L2 layer equipment is not optimized in the leased network. 
The experiment results show that, in addition to tuning kernel-level parameters at the system level, end-to-end servers with coordinated elements can take advantage of the available bandwidth.

Tan et al. [23] proposed TSDEGA (time synchronization and enhanced greedy algorithm based on $\mathrm{D}(\mathrm{v})$ ), a routing algorithm suitable for structural health monitoring. The proposed algorithm can identify whether the node has been accessed by setting the access marker based on the existing greedy algorithm to solve the node coverage problem and the degree of the node. As a result, each node can only be accessed once in the same round, thereby significantly reducing time and complexity. The proposed algorithm improves the stability of time synchronization and synchronization accuracy and eliminates the interference of outliers while maintaining the original lower communication overhead.

Park et al. [24] described the advanced proposal for the transportation management plan (TMP) development of expressway work zones. This study analyzed the association between lane closure types of expressway work zones and the types of operations performed, using text mining techniques for large-scale traffic data sets with descriptive text. The relationship between work types and lane closure types was investigated using text mining techniques and big data analysis for massive data sets in expressway work zones. The aim is to improve the overall efficiency of TMP by examining the unique patterns implied in these relationships, through the identification of the common characteristics of various work zones and utilization of proper applications based on the targeted TMP strategies.

Acknowledgements I appreciate Prof. Neil Yen who had helped me manage this special section.

\section{References}

1. Xu L, Weng CY, Yuan LP et al (2015) A shareable keyword search over encrypted data in cloud computing. J Supercomput. https://doi.org/10.1007/s11227-015-1515-8

2. Nkenyereye L, Park Y, Rhee KH (2016) Secure vehicle traffic data dissemination and analysis protocol in vehicular cloud computing. J Supercomput. https://doi.org/10.1007/s11227-016-1773-0

3. Chen M, Hao Y, Li Y, Lai C, Wu D (2015) On the computation offloading at ad hoc cloudlet: architecture and service modes. IEEE Commun Mag 53(6):18-24

4. Chen L, Qiu M, Song J et al (2016) E2FS: an elastic storage system for cloud computing. J Supercomput. https://doi.org/10.1007/s11227-016-1827-3

5. Muñoz FR, Villalba LJG (2016) An algorithm to find relationships between web vulnerabilities. J Supercomput. https://doi.org/10.1007/s11227-016-1770-3

6. Lim J, Jeong YS, Park DS et al (2016) An efficient distributed mutual exclusion algorithm for intersection traffic control. J Supercomput. https://doi.org/10.1007/s11227-016-1799-3

7. Al-Sultan S, Al-Doori MM, Al-Bayatti AH, Zedan H (2014) A comprehensive survey on vehicular ad hoc network. J Netw Comput Appl 37:380-392

8. Whaiduzzaman M, Sookhak M, Gani A, Buyya R (2014) A survey on vehicular cloud computing. J Netw Comput Appl 40:325-344. https://doi.org/10.1016/j.jnca.2013.08.004

9. Ji S, Tan C, Yang P et al (2016) Compressive sampling and data fusion based structural damage monitoring in wireless sensor network. J Supercomput. https://doi.org/10.1007/s11227-016-1938-X

10. Goi MT, Kalidas V, Zeeshan M (2014) Comparison of stimulus-organism-response framework between international and local retailer. Procedia Soc Behav Sci 130:461-468

11. Gabus A, Fontela E (1972) World problems, an invitation to further thought within the framework of DEMATEL. Battelle Geneva Research Center, Geneva 
12. Opricovic S, Tzeng GH (2004) Compromise solution by MCDM methods: a comparative analysis of VIKOR and TOPSIS. Eur J Oper Res 156(2):445-455

13. Chen YS, Wu C, Chu HH et al (2017) Analysis of performance measures in cloud-based ubiquitous SaaS CRM project systems. J Supercomput. https://doi.org/10.1007/s11227-017-1978-X

14. Ramadoss R, Elango NM, Abimannan S et al (2017) Non-intrusive transaction aware filtering during enterprise application modernization. J Supercomput. https://doi.org/10.1007/s11227-017-2123-6

15. Lee KM, Lee KM, Lee SH (2017) Remote data integrity check for remotely acquired and stored stream data. J Supercomput. https://doi.org/10.1007/s11227-017-2117-4

16. Hao F, Min G, Pei Z, Park DS, Yang LT (2017) K-clique Communities Detection in Social Networks based on Formal Concept Analysis. IEEE Syst J 11(1):250-259

17. Hao F, Park DS (2017) cSketch: a novel framework for capturing cliques from big graph. J Supercomput. https://doi.org/10.1007/s11227-017-2114-7

18. Kim HY (2017) A load balancing scheme with loadbot in IoT networks. J Supercomput. https://doi. org/10.1007/s11227-017-2087-6

19. Min S, Jeong Y, Kang J (2017) Cross-layer design and performance analysis for maximizing the network utilization of wireless mesh network in cloud computing. J Supercomput. https://doi.org/10. 1007/s11227-017-2146-Z

20. Alexey G, Parygin DS, Finogeev AA (2017) The convergence computing model for big sensor data mining and knowledge discovery. Hum Centric Comput Inf Sci 7:11

21. Vogel A, Griebler D, Schepke C, Fernandes LG (2017) An intra-cloud networking performance evaluation on cloudstack environment. In: Proceedings of PDP

22. Lee WH, Park JS, Kim SH et al (2017) A method for enhancing end-to-end transfer efficiency via performance tuning factors on dedicated circuit networks with a public cloud platform. J Supercomput. https://doi.org/10.1007/s11227-017-2157-9

23. Tan C, Ji S, Gui Z et al (2017) An effective data fusion based routing algorithm with time synchronization support for vehicular wireless sensor networks. J Supercomput. https://doi.org/10.1007/s11227017-2145-0

24. Park SH, Synn J, Kwon OH et al (2017) Apriori-based text mining method for the advancement of the transportation management plan in expressway work zones. J Supercomput. https://doi.org/10.1007/ s11227-017-2142-3 